\title{
Kasuistiken
}

Ophthalmologe $2021 \cdot 118: 838-841$ https://doi.org/10.1007/s00347-020-01202-6 Online publiziert: 15 . August 2020

(c) Der/die Autor(en) 2020

M. Anwar $(\mathbb{D} \cdot$ T. Brockmann - M. Walckling - T. A. Fuchsluger

Klinik und Poliklinik für Augenheilkunde, Universitätsmedizin Rostock, Rostock, Deutschland

\section{Akutes Engwinkelglaukom und Effusionssyndrom nach Phakoemulsifikation}

\section{Anamnese}

Eine 72-jährige Patientin stellte sich in unserer Klinik als Notfall mit starken Kopfschmerzen, Übelkeit und Erbrechen sowie beidseitig plötzlicher Sehverschlechterung vor. Am späten Vormittag des Tages erhielt sie an ihrem rechten Auge eine elektive ambulante Kataraktoperation unter Lokalanästhesie. Die Kataraktoperation des Partnerauges erfolgte bereits 2 Wochen zuvor. An beiden Augen verliefen die Operationen komplikationslos mit Implantation
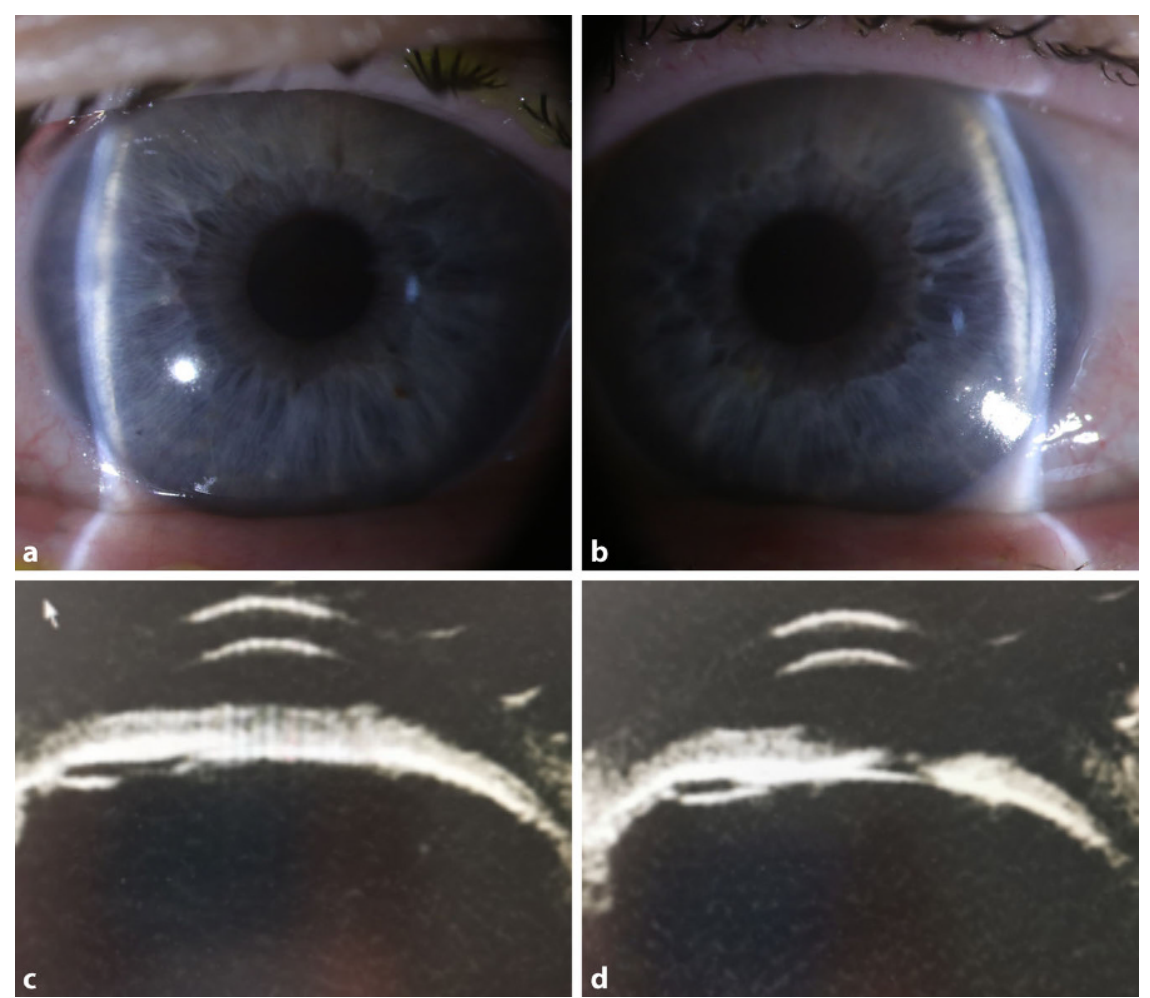

Abb. 1 A Spaltlampenmikroskopie des rechten (a) und linken (b) Auges nach initialer Drucksenkung mit flacher Vorderkammer. Die Ultraschallbiomikroskopie des vorderen rechten (c) und linken (d) Augenabschnitts zeigt die Anteflexion des Iris-Intraokularlinse-Diaphragmas mit iridokornealem Winkelblock

Kapselsack. Postoperativ wurde der Patientin, neben der antibiotischen und antiinflammatorischen Lokaltherapie eine prophylaktische orale Einmaldosis Acetazolamid $250 \mathrm{mg}$ verordnet, um einem möglichen postoperativen intraokularen Druckanstieg vorzubeugen. Die Patientin nahm die AcetazolamidTablette $4 \mathrm{~h}$ nach der Operation ein und legte sich schlafen. Etwa 6 h später wachte sie aufgrund starker Kopfschmerzen auf und bemerkte beidseits eine massive 
Sehverschlechterung, woraufhin sie die Klinik aufsuchte.

\section{Klinischer Befund}

Bei der Erstvorstellung betrug die bestkorrigierte Sehschärfe der Patientin rechts $1 / 35$ und links $1 / 25$ bei einer Refraktionskorrektur rechts von $-3,0 \mathrm{dpt}$ und links $-3,5 \mathrm{dpt}$. Spaltlampenmikroskopisch zeigten sich beidseits eine Bindehautinjektion, ausgeprägtes Hornhautödem sowie eine flache Vorderkammer mit Anteflexion der Intraokularlinse (• Abb. 1a, b). Zur weiteren Diagnostik führten wir eine Ultraschallbiomikroskopie durch (- Abb. 1c, d). Hierbei betrug die Vorderkammertiefe rechts $2,08 \mathrm{~mm}$ und links $2,74 \mathrm{~mm}$, der Kammerwinkel zeigte sich bei einem iridokornealen Winkelblock zirkulär verschlossen. Der Intraokulardruck betrug initial rechts $62 \mathrm{mmHg}$ und links $64 \mathrm{~mm} \mathrm{Hg}$. Eine Fundoskopie war aufgrund des ausgeprägten Hornhautödems nicht möglich. In der B-ScanSonographie zeigte sich beidseits eine Aderhautschwellung im Sinne einer Aderhautabhebung (- Abb. 2c, d). Die Patientin wurde mit der Arbeitsdiagnose sekundäres Engwinkelglaukom mit Pupillarblock stationär aufgenommen.

\section{Therapie und Verlauf}

Eine initiale systemisch-intravenöse Drucksenkung erfolgte mit Acetazolamid $500 \mathrm{mg}$ und Mannitol (15\%) $300 \mathrm{ml}$ sowie beidseits lokal mit Timolol 0,5\%, Apraclonidin 5\% und Prednisolon 1\%. Unter der genannten Therapie zeigte sich nur eine unzureichende Drucksenkung bei Zunahme der Aderhautschwellung, sodass die Arbeitsdiagnose überdacht werden musste. Acetazolamid wurde im Weiteren als Ursache für ein bilaterales uveales Effusionssyndrom mit sekundärem iridokornealem Engwinkelglaukom angenommen und daher abgesetzt. Dementsprechend erfolgte die weitere systemische Therapie mit einer erneuten Einmalgabe von Mannitol (15\%) $300 \mathrm{ml}$ intravenös und Prednisolon $100 \mathrm{mg}$ per os täglich. Hierunter verbesserte sich der klinische Befund über 3 Tage allmählich. Der Augeninnendruck konnte

Ophthalmologe 2021 · 118:838-841 https://doi.org/10.1007/s00347-020-01202-6

(c) Der/die Autor(en) 2020

\section{Anwar · T. Brockmann · M. Walckling · T. A. Fuchsluger}

\section{Akutes Engwinkelglaukom und Effusionssyndrom nach Phakoemulsifikation}

\section{Zusammenfassung}

Eine 72-jährige Patientin entwickelte nach unkomplizierter Kataraktoperation ein beidseitiges sekundäres iridokorneales Engwinkelglaukom bei uvealem Effusionssyndrom. Als Ursache für die Entstehung des Effusionssyndroms konnte die postoperative Einnahme von Acetazolamid ausgemacht werden. Unter Berücksichtigung einer Sulfonamid-freien systemischen und lokalen drucksenkenden sowie antiinflammatorischen Therapie zeigte sich eine schnelle
Befundbesserung. Der Fall verdeutlicht eine seltene, aber klinisch schwerwiegende unerwünschte Arzneimittelwirkung von Acetazolamid und zeigt effiziente Therapiemöglichkeiten auf.

Schlüsselwörter Acetazolamid - Uveales Effusionssyndrom . Sulfonamid-Allergie - Kataraktoperation . Unerwünschte Arzneimittelwirkung (UAW)

\section{Acute angle-closure glaucoma and effusion syndrome after phacoemulsification}

Abstract

A 72-year-old female patient developed bilateral secondary iridocorneal angle-closure glaucoma with uveal effusion syndrome after uncomplicated cataract surgery. The postoperative intake of acetazolamide was identified as causative for the development of the effusion syndrome. Taking a sulfonamidefree systemic and local intraocular pressure lowering and anti-inflammatory treatment into account, a rapid improvement of the ocular manifestation was achieved. The case illustrates a rare but clinically severe adverse effect of acetazolamide and outlines efficient treatment options.

Keywords

Acetazolamide - Uveal effusion syndrome .

Sulfonamide allergy · Cataract surgery .

Adverse drug reaction rechts auf $7 \mathrm{~mm} \mathrm{Hg}$ und links $9 \mathrm{~mm} \mathrm{Hg}$ gesenkt werden. In der Fundoskopie zeigte sich noch eine bilaterale periphere Aderhautabhebung (• Abb. 2a, b). Der bestkorrigierte Dezimalvisus stieg auf rechts 0,8 und links 0,9 an, die Konfiguration der Vorderkammer normalisierte sich, und die Myopisierung zeigte sich rückläufig. Die systemische und lokal drucksenkende Therapie wurde beendet.

In der Verlaufskontrolle zeigte sich nach 1 Woche beidseits ein regelrechter sonographischer und fundoskopischer Befund des hinteren Augenabschnitts (• Abb. 3). Die Vorderkammertiefe betrug rechts $4,39 \mathrm{~mm}$ und links $4,37 \mathrm{~mm}$ bei einem Augeninnendruck von rechts $10 \mathrm{mmHg}$ und links $11 \mathrm{mmHg}$. Der unkorrigierte Dezimalvisus betrug nun beidseits 1,0 . In der nochmals durchgeführten Ultraschallbiomikroskopie of- fenbarte sich nun beidseits eine PlateauIris-Konfiguration ( $\bullet$ Abb. 3c, d).

\section{Diagnose}

Beidseitiges akutes Engwinkelglaukom bei Acetazolamid-indiziertem Effusionssyndrome nach Kataraktoperation in Plateau-Iris-Konfiguration.

\section{Diskussion}

Die Entwicklung eines uvealen Effusionssyndroms ist bei Sulfonamid-haltigen Arzneimitteln, zu denen auch der Carboanhydrasehemmer Acetazolamid gehört, grundsätzlich bekannt [9]. Der vorliegende Fall verdeutlicht allerdings, dass die hierbei eintretende Aderhautschwellung nicht nur Aderhautfalten hervorruft, sondern foudroyant verlaufen und dabei durch eine massive intraokulare 

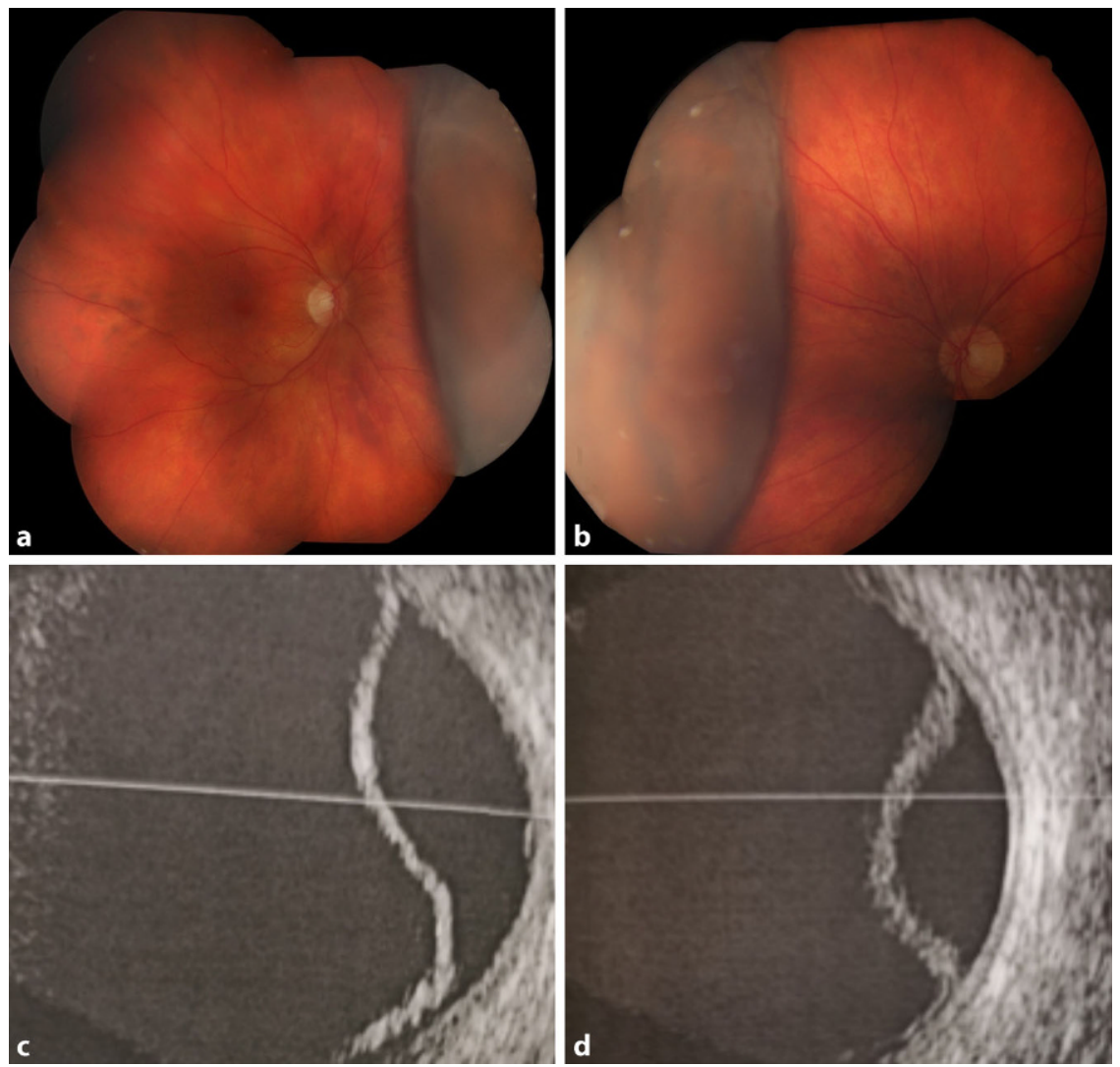

Abb. $2 \Delta$ B-Scan-Sonographie des hinteren Augenabschnitts des rechten (c) und linken (d) Auges mit deutlicher Aderhautabhebung vor initialer Drucksenkung. Der rechte (a) und linke (b) Fundusbefund zeigten nach initialer Drucksenkung eine bilaterale periphere nasale Aderhautabhebung

Volumenverschiebung zu einem iridokornealen Winkelblock führen kann. Neben Acetazolamid sind aus der Literatur ähnliche Fälle auch durch andere Sulfonamid-haltige Medikamente wie dem als Antiepileptikum verwendeten Carboanhydrasehemmer Topiramat und dem Thiaziddiuretikum Hydrochlorothiazid bekannt $[4,7,8]$. In der Allgemeinbevölkerung wird die Inzidenz von Sulfonamid-Allergien mit 3-8\% angenommen [2]. Die meisten Reaktionen auf Sulfonamide resultieren aus multifaktoriellen immunologischen und toxischen Stoffwechselmechanismen, während über die genauen Reaktionsmechanismen weniger bekannt ist [1]. Immunglobulin $\mathrm{E}(\mathrm{IgE})$-vermittelte Typ1-Immunreaktionen auf Sulfonamide können Anaphylaxie, Angioödeme und Urtikaria hervorrufen und nehmen darüber auch Einfluss auf die Konstitution der Aderhaut [10]. Über diesen Weg kann u. a. Acetazolamid, wie in dieser Kasuistik geschildert, paradoxe Nebenwirkungen mit ausgeprägtem uvealem Effusionssyndrom hervorrufen. Interessanterweise wurde festgestellt, dass gerade bei IgEvermittelten Typ-1-Immunreaktionen nicht die Sulfonamid-definierende $\mathrm{NH}_{2}$ $\mathrm{SO}_{2}$-Struktur, sondern der im Molekül enthaltene heterozyklische N-Ring an IgE bindet [3]. Somit ist auch zu erklären, dass verschiedene Sulfonamidhaltige Arzneimittel mit unterschiedlichen heterozyklischen N-Ringen sehr variable Immunreaktionen hervorrufen können. In dem vorliegenden Fall war bei der Patientin keine Sulfonamid-Allergie bekannt. Dennoch sollte gerade vor der Verabreichung von Acetazolamid und anderen Sulfonamiden eine allergologische Anamnese erfolgen. Gleichzeitig sollte auch bei Vorliegen einer klaren klinischen Situation wie der eines Winkelblocks durch eine spezifische Anamnese differenzialdiagnostisch an die Unterscheidung zwischen einem primären und sekundären Engwinkelglaukom gedacht werden, da dies für die Therapieentschei- dung - wie in diesem Fall - von wesentlicher klinischer Relevanz sein kann. In diesem Kontext wurde in der Literatur das Vorliegen einer Plateau-Iris-Konfiguration als wesentlicher Risikofaktor für die Entwicklung eines Winkelblockglaukoms diskutiert [5]. Während lokale und/ oder systemische Carboanhydrasehemmer im Rahmen von Augeninnendruckentgleisungen als First-line-Therapeutika angesehen und verwendet werden können [6], wären diese in dem vorliegenden Fall kontraindiziert. Nicht zuletzt sollte neben der adäquaten Therapie auch daran gedacht werden, den Patienten über das Vorliegen und die Bedeutung einer Sulfonamid-Allergie aufzuklären.

\section{Fazit für die Praxis}

\section{- Ein beidseitiges sekundäres irido-} korneales Engwinkelglaukom auf Grundlage eines uvealen Effusionssyndroms kann als seltene, klinisch schwerwiegende unerwünschte Arzneimittelwirkung von Acetazolamid eintreten.

- Die Verordnung von Acetazolamid sollte mit Bedacht erfolgen, und mögliche Sulfonamid-Allergien müssen berücksichtigt werden.

- Eine effiziente und sichere Möglichkeit der Akutbehandlung besteht in der Verwendung von Osmodiuretika und Kortikosteroiden.

\section{Korrespondenzadresse

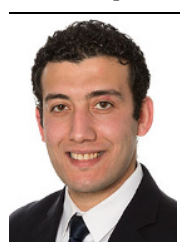 \\ M. Anwar \\ Klinik und Poliklinik für Augenheilkunde, Universitätsmedizin Rostock Doberaner Str. 140, 18057 Rostock, Deutschland mahmoud.anwar@ med.uni-rostock.de}

Funding. Open Access funding provided by Projekt DEAL.

\section{Einhaltung ethischer Richtlinien}

Interessenkonflikt. M. Anwar, T. Brockmann, M. Walckling und T.A. Fuchsluger geben an, dass kein Interessenkonflikt besteht.

Für diesen Beitrag wurden von den Autoren keine Studien an Menschen oder Tieren durchgeführt. Für 

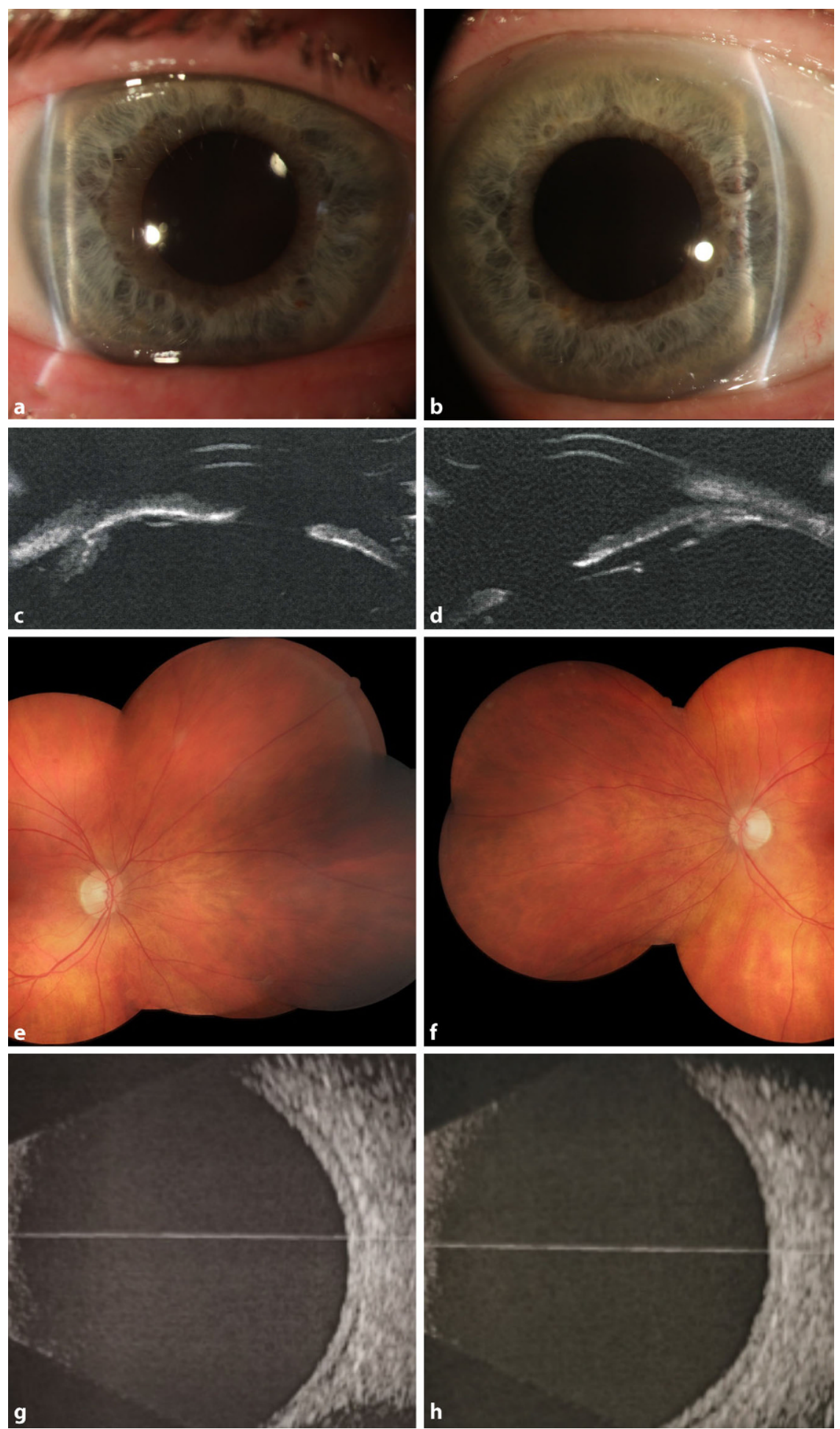

Abb. 3 A Befund in der Verlaufskontrolle nach 10 Tagen. Spaltlampenmikroskopie des rechten (a) und linken (b) Auges mit tiefer Vorderkammerkonfiguration. In der Ultraschallbiomikroskopie des vorderen rechten (c) und linken (d) Augenabschnitts offenbart sich eine Plateau-Iris. Am hinteren Augenabschnitt zeigt sich die Aderhaut, Befund entsprechend am rechten und linken Auge, in der Fundoskopie $(\mathbf{e}, \mathbf{f})$ sowie in der B-Scan-Sonographie $(\mathbf{g}, \mathbf{h})$ flach anliegend die aufgeführten Studien gelten die jeweils dort angegebenen ethischen Richtlinien. Für Bildmaterial oder anderweitige Angaben innerhalb des Manuskripts, über die Patienten zu identifizieren sind, liegt von innen und/oder ihren gesetzlichen Vertretern eine schriftliche Einwilligung vor.

Open Access Dieser Artikel wird unter der Creative Commons Namensnennung 4.0 International Lizenz veröffentlicht, welche die Nutzung, Vervielfältigung, Bearbeitung, Verbreitung und Wiedergabe in jeglichem Medium und Format erlaubt, sofern Sie den/die ursprünglichen Autor(en) und die Quelle ordnungsgemäß nennen, einen Link zur Creative Commons Lizenz beifügen und angeben, ob Änderungen vorgenommen wurden.

Die in diesem Artikel enthaltenen Bilder und sonstiges Drittmaterial unterliegen ebenfalls der genannten Creative Commons Lizenz, sofern sich aus der Abbildungslegende nichts anderes ergibt. Sofern das betreffende Material nicht unter der genannten Creative Commons Lizenz steht und die betreffende Handlung nicht nach gesetzlichen Vorschriften erlaubt ist, ist für die oben aufgeführten Weiterverwendungen des $\mathrm{Ma}$ terials die Einwilligung des jeweiligen Rechteinhabers einzuholen.

Weitere Details zur Lizenz entnehmen Sie bitte der Lizenzinformation auf http://creativecommons.org/ licenses/by/4.0/deed.de.

\section{Literatur}

1. Dibbern DA, Montanaro A (2008) Allergies to sulfonamide antibiotics and sulfur-containing drugs. Ann Allergy Asthma Immunol 100:91-100 (quiz 100-103,111)

2. Giles A, Foushee J, Lantz E et al (2019) Sulfonamide Allergies. Pharmacy (Basel). https://doi.org/10. 3390/pharmacy7030132

3. Harle DG, Baldo BA, Wells JV (1988) Drugs as allergens: detection and combining site specificities of $\mathrm{lgE}$ antibodies to sulfamethoxazole. Mol Immunol 25:1347-1354

4. Malagola R, Arrico L, Giannotti R et al (2013) Acetazolamide-induced cilio-choroidal effusion after cataract surgery: unusual posterior involvement. Drug Des Devel Ther 7:33-36

5. Man X, Costa R, Ayres BM et al (2016) Acetazolamide-induced bilateral ciliochoroidal effusion syndrome in plateau iris configuration. Am J Ophthalmol 3:14-17 (Case Rep)

6. Mincione F, Scozzafava A, Supuran CT (2008) The development of topically acting carbonic anhydrase inhibitors as antiglaucoma agents. Curr Pharm Des 14:649-654

7. Parthasarathi S, Myint K, Singh G et al (2007) Bilateral acetazolamide-induced choroidal effusion following cataract surgery. Eye (Lond) 21:870-872

8. Roh YR, Woo SJ, Park KH (2011) Acute-onset bilateral myopia and ciliochoroidal effusion induced by hydrochlorothiazide. Korean J Ophthalmol 25:214-217

9. Senthil S, Garudadri C, Rao HB et al (2010) Bilateral simultaneous acute angle closure caused by sulphonamide derivatives: a case series. Indian J Ophthalmol 58:248-252

10. Wulf NR, Matuszewski KA (2013) Sulfonamide cross-reactivity: is there evidence to support broad cross-allergenicity? Am J Health Syst Pharm 70:1483-1494 